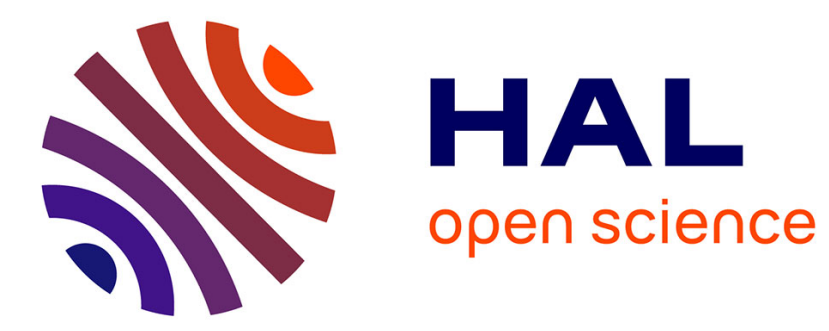

\title{
Synergistic impeding of phonon transport through resonances and screw dislocations
}

Hongying Wang, Yajuan Cheng, Masahiro Nomura, Sebastian Volz, Davide Donadio, Xiaohong Zhang, Shiyun Xiong

\section{- To cite this version:}

Hongying Wang, Yajuan Cheng, Masahiro Nomura, Sebastian Volz, Davide Donadio, et al.. Synergistic impeding of phonon transport through resonances and screw dislocations. Physical Review B, 2021, 103, 10.1103/physrevb.103.085414 . hal-03420109

\section{HAL Id: hal-03420109 https://hal.science/hal-03420109}

Submitted on 9 Nov 2021

HAL is a multi-disciplinary open access archive for the deposit and dissemination of scientific research documents, whether they are published or not. The documents may come from teaching and research institutions in France or abroad, or from public or private research centers.
L'archive ouverte pluridisciplinaire HAL, est destinée au dépôt et à la diffusion de documents scientifiques de niveau recherche, publiés ou non, émanant des établissements d'enseignement et de recherche français ou étrangers, des laboratoires publics ou privés. 


\title{
Synergistic impeding of phonon transport through resonances and screw dislocations
}

\author{
Hongying Wang, ${ }^{1}$ Yajuan Cheng,,${ }^{2,}$ Masahiro Nomura, ${ }^{3}$ Sebastian Volz,,${ }^{3,4}$ \\ Davide Donadio, ${ }^{5}$ Xiaohong Zhang, ${ }^{1, \dagger}$ and Shiyun Xiong ${ }^{1,3,+}$ \\ ${ }^{1}$ Institute of Functional Nano and Soft Materials (FUNSOM), Jiangsu Key Laboratory for Carbon-Based Functional Materials and Devices, \\ Soochow University, 199 Ren'ai Road, Suzhou, 215123 Jiangsu, People's Republic of China \\ ${ }^{2}$ Key Laboratory of Organic Synthesis of Jiangsu Province and the State and Local Joint Engineering Laboratory for Novel Functional \\ Polymeric Materials, College of Chemistry, Chemical Engineering and Materials Science, Soochow University, \\ Suzhou 215123, People's Republic of China \\ ${ }^{3}$ Institute of Industrial Science, The University of Tokyo, Tokyo 153-8505, Japan \\ ${ }^{4}$ Laboratory for Integrated Micro Mechatronic Systems (LIMMS/CNRS-IIS), The University of Tokyo, Tokyo 153-8505, Japan \\ ${ }^{5}$ Department of Chemistry, University of California, Davis, One Shields Avenue, Davis, California 95616, USA
}

(Received 24 October 2020; revised 9 January 2021; accepted 19 January 2021; published 9 February 2021)

\begin{abstract}
Improving the control of heat flow at the nanoscale is essential for promoting its applications in many fields, such as energy conversion, thermal informatics, and communication technologies. Here we perform a systematic study on the synergistic effect of screw dislocations and surface resonators on thermal transport of $\mathrm{Si}$ nanowires and the corresponding mechanisms based on molecular dynamics simulations. We uncover that screw dislocations reduce the thermal conductivity by enhancing the anharmonicity of nanowires due to the nonhomogeneous stress field. For resonant structures, we demonstrate that the suppression of relaxation time is the main mechanism for thermal conductivity reduction. The suppression of relaxation time by more than two orders of magnitude below $4 \mathrm{THz}$ dramatically reduces the resonant structure thermal conductivity, while the previously proposed group velocity reduction mechanism can only impede phonon transport beyond $4 \mathrm{THz}$ slightly. By comparing the mechanisms produced by dislocations and resonators, we find that the resonators have a stronger effect over screw dislocations in impeding the phonon transport at low frequencies while it becomes opposite at high frequencies. As a result, they can be combined together to manipulate phonon transport synergistically at all frequencies. Our findings not only provide insights into the mechanisms of thermal conductivity engineering by screw dislocations and surface resonators, but they also illustrate a paradigm for ultralow thermal conductivity design through the tailoring of the entire frequency range of phonon transport.
\end{abstract}

DOI: 10.1103/PhysRevB.103.085414

\section{INTRODUCTION}

Engineering thermal transport in materials has received a tremendous amount of attention during the past decades due to its wide applications, ranging from energy conversion to information and communication technologies. To enable a highly efficient control of heat flow, it is essential to manipulate phonon transport over a broad frequency range. Traditionally, phonon transport has been widely engineered by phonon scattering [1-4], i.e., by introducing scattering centers inside materials or reducing dimensionality [5]. This strategy has achieved great success in decreasing the thermal conductivity (TC) of semiconductors, especially in promoting the development of thermoelectric materials [6], where low TC and high electrical conductivity are preferable simultaneously. Phonon scattering is currently based on the phonon particle picture described by the Boltzmann transport equation (BTE), and it is efficient in impeding

\footnotetext{
*yjcheng@suda.edu.cn

†xiaohong_zhang@suda.edu.cn

${ }^{\ddagger}$ xiongshiyun216@163.com
}

the transport of high-frequency phonons. However, due to the long-wavelength feature of low-frequency modes, it is difficult to hinder low-frequency phonon transport by scattering. To enable efficient transport control of low-frequency phonons, the phonon resonant mechanism was introduced recently. Relevant research on semiconductor materials, such as silicon nanowires (NWs) and membranes, carbon nanotubes, and graphene nanoribbons, remarkably verify tunable TC arising from the resonant phonon hybridization, especially for the effect on low-frequency phonons [7-14]. In general, all the structures that can produce flat bands in part of or in the entire Brillouin zone can be regarded as resonant structures. Such structures can be formed with confined atom motions or confined phonon propagation in at least one direction. The former includes caged structures such as skutterudite $[15,16]$ or clathrates $[17,18]$. The latter may consist of systems with surface pillared structures $[7,8,19]$. Usually, the structures with confined atoms in lattices have a limited number of resonant modes due to the small number of degrees of freedom associated with the motion of the caged atoms. In contrast, the surface resonant structures can provide a large number of resonant modes in the entire frequency range through a tailored design of the resonant structures. This entails a 
substantial advantage of the surface resonant structure in TC engineering.

Different from phonon scattering centers, the surface resonant structures, which can produce numerous standing waves in the entire frequency range, are located on the surface of materials [8]. Surface nanostructures have a minimal effect on electrical conductivity, thus providing a promising route to engineer better thermoelectric materials. The produced resonant modes (standing waves in resonant structures) can hybridize with the propagating modes by altering their dispersion relations and reducing their group velocities $v_{g}$. This mechanism has been widely accepted as the main explanation for the effect of resonances on the TC of NWs, thin films, and membranes $[8,20]$. However, through phonon mean free path (MFP) calculations, it was found in our previous work $[8,21]$ that the low-frequency $(0-2 \mathrm{THz})$ phonon MFP can be reduced by more than two orders of magnitude, while the averaged phonon group velocity is only reduced up to five times, which is insufficient to reproduce the obtained MFP. This discrepancy between phonon MFP and group velocity reduction indicates that the latter is not the only effect of resonances. Since the phonon MFP is given by the product $\Lambda=v_{g} \tau$, where $\tau$ is the phonon relaxation time, $\tau$ must also be affected by resonances. Therefore, for a better design of resonant structures, and to promote their applications, it is necessary to investigate further the detailed mechanisms of resonances and their effect on phonon transport in nanostructures.

Moreover, it was revealed that the resonant mechanism is less effective in impeding the transport of high-frequency phonons $[8,21]$. As a result, it needs to be combined with scattering, so as to efficiently hinder phonon transport over the entire frequency range. With the advances of modern nanowire growth technology, dendritic Si NWs, grown by screw dislocations, have been synthesized experimentally [22]. These structures consist of NWs with surrounding pillars and a screw dislocation in the middle. In such systems, the surrounding branches can act as resonant structures, while the central screw dislocation can be treated as a source of enhanced phonon scattering. As a result, screw dislocated dendritic NWs are ideal platforms for studying the synergistic effect between scattering and resonant mechanisms. Different from other scattering sources (e.g., point defects or interfaces), dislocations do not scatter phonons directly, but they are hypothesized to enhance lattice anharmonicity $[23,24]$. However, the link between dislocations and increased anharmonicity has not been unveiled yet, and it is not known which phonon frequencies are most affected by dislocations.

In this work, we address the effects of surface resonances, screw dislocations, and the combination of the two in silicon NWs by extensive equilibrium molecular dynamics (EMD) simulations. We study four types of Si NWs: pristine NWs, screw dislocated (SD) NWs, resonant (Re) NWs, and screw dislocated resonant (SDRe) NWs. The SD NW, Re NW, and SDRe NW structures proposed in our work can be synthesised through the vapor-liquid-solid or/and dislocation-driven methods [22,25-28]. In this work, we investigate the synergistic reduction of TC in nanostructures by dislocations and branches as well as the corresponding mechanisms. Our simulations indicate that the TC of SDRe NWs can reach a

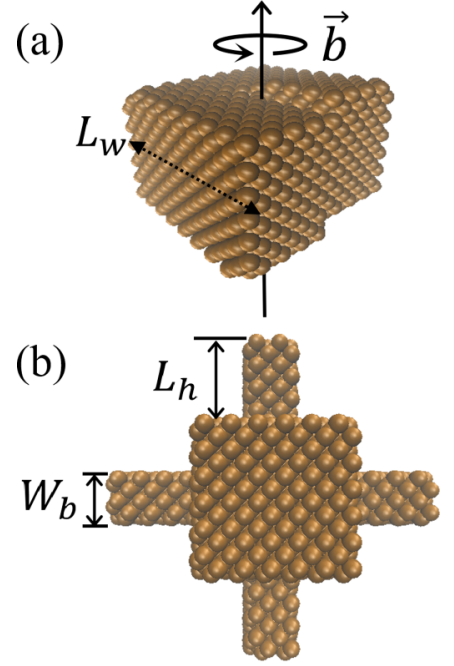

(c)

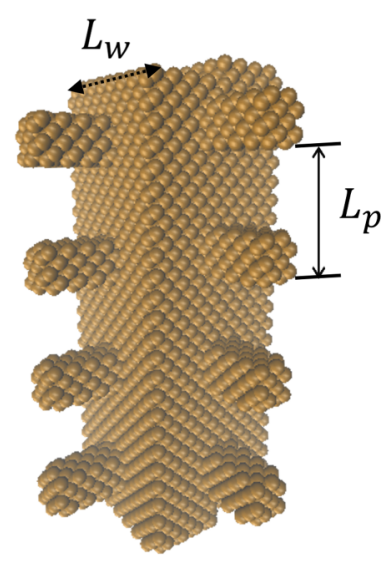

FIG. 1. (a) Side view of a SD NW with Burgers vector $\vec{b}=$ $0.54 \mathrm{~nm}$; (b) top view of a Re NW; and (c) side view of a SDRe NW. Here $L_{w}$ represents the main NW cross-section width, $L_{h}$ and $W_{b}$ are the height and the width of resonant branches, and $L_{p}$ is the periodic length of resonant structures.

maximum reduction of $70 \%$ compared to one of the pristine structures, which is $27 \%$ and $10 \%$ larger than the reductions solely due to dislocations and resonances, respectively. The resonant structures, besides reducing the group velocities, diminish the relaxation times of low-frequency phonons by more than two orders of magnitude, which is much larger than the relaxation time reduction produced by dislocations. Moreover, we find that the inhomogeneous stress, introduced by screw dislocations, enhances lattice anharmonicity over the whole phonon spectrum, thus leading to the observed TC reduction, especially for the contribution of low-frequency modes.

\section{METHODS}

We constructed models of square-section silicon NWs, grown along the $\langle 100\rangle$ crystallographic direction with a different cross-section width $L_{w}$. A screw dislocation with Burgers vector $\vec{b}$ is then introduced in the middle of the NW and/or pillars are attached on the four surfaces (Fig. 1). The crosssection width $\left(W_{b}\right)$, the height $\left(L_{h}\right)$, and the period length $\left(L_{p}\right)$ of resonant pillars are fixed as $1.09,2.17$, and $3.26 \mathrm{~nm}$, respectively. The length of all NWs is $13.0 \mathrm{~nm}$, which is sufficient to attain well converged TC according to our test. All simulations are performed with LAMMPS [29] with the interatomic interactions described by the Stillinger-Weber three-body potential [30], which is commonly adopted for TC calculations in silicon-based materials $[11,31,32]$. The integration time step is set to $0.5 \mathrm{fs}$, which is small enough to ensure energy conservation. All systems are first equilibrated to standard ambient conditions $(T=300 \mathrm{~K}$ and $P=1 \mathrm{~atm})$ in the NPT ensemble using the Nosé-Hoover thermostat and barostat $[33,34]$ for $8 \mathrm{~ns}$, followed by an $N V T$ equilibration for 4 ns. Eventually, the systems are evolved in the $N V E$ ensemble for $7 \mathrm{~ns}$ with a heat flux collection every $10 \mathrm{fs}$ for the last $5 \mathrm{~ns}$. The TC is then calculated based on the Green-Kubo 


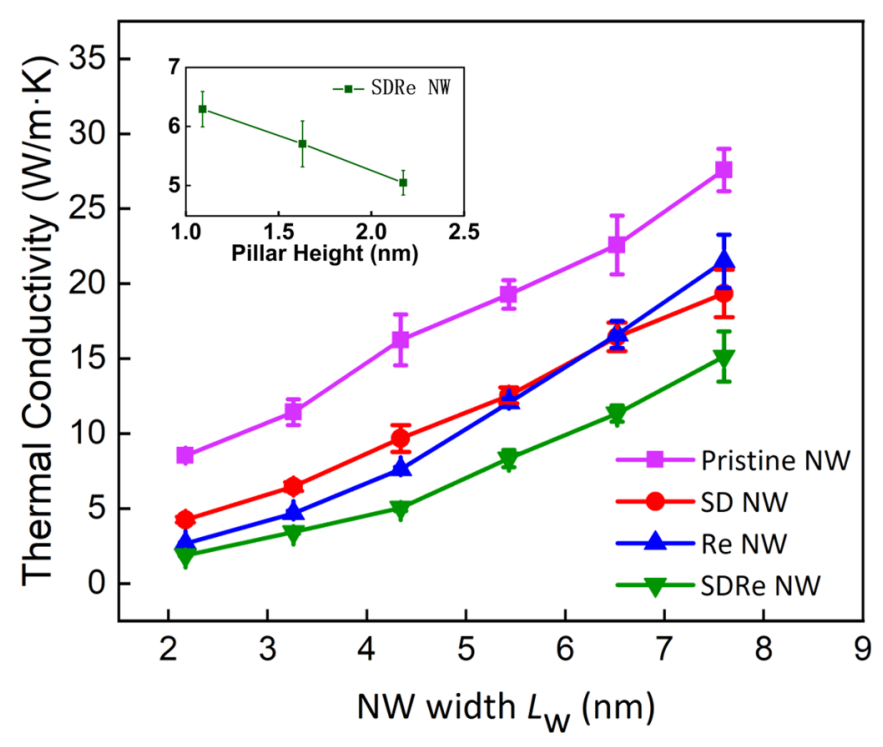

FIG. 2. Thermal conductivity as a function of NW cross-section width $L_{w}$ for pristine, SD, Re, and SDRe NWs. The inset shows the thermal conductivity of SDRe NWs with $L_{w}=4.34 \mathrm{~nm}$ vs the height of the pillars.

formula [35-37] with 24 ensemble averages starting from different initial velocities. As the definition of the cross-section of branched systems is somewhat arbitrary, to compare to the results in Ref. [11], the volume of the entire structure including the pillars is taken into account for the Green-Kubo TC calculations of pillared NWs. Further simulations are carried out at higher temperatures, up to $600 \mathrm{~K}$, to unravel the temperature dependence of TC in different NWs. Molecular dynamics simulations are based on classical mechanics, which results in a Boltzmann distribution of phonons populations, which converges to the Bose-Einstein distribution beyond the Debye temperature $(645 \mathrm{~K})$. In this work, the temperature correction due to the quantum effect [38] is not considered, as the procedure to rescale the temperature has been proven incorrect, and it would not affect relative TC trends among different structures and our conclusions [39].

\section{RESULTS AND DISCUSSIONS}

The effect of screw dislocations and resonances on the TC of NWs is first examined and the corresponding results are illustrated in Fig. 2. To validate our simulation approach, we first computed the TC of bulk Si, which results in $388 \pm$ $31 \mathrm{~W} \mathrm{~m}^{-1} \mathrm{~K}^{-1}$ at room temperature. This value is close to the value of $376 \pm 19 \mathrm{~W} \mathrm{~m}^{-1} \mathrm{~K}^{-1}$ of Ref. [11]. The TC of the pristine NW with $L_{w}=4.34 \mathrm{~nm}$ in our simulations is about $16.4 \pm 0.9 \mathrm{~W} \mathrm{~m}^{-1} \mathrm{~K}^{-1}$ at $300 \mathrm{~K}$, which is also close to that obtained in [40] $\left(19.7 \mathrm{~W} \mathrm{~m}^{-1} \mathrm{~K}^{-1}\right)$ based on nonequilibrium molecular dynamics simulations (NEMD). The deviation might originate from the differences between NEMD and EMD simulations. It is also worth noting that the TC obtained in our NWs bounded with $\{100\}$ surfaces is smaller than the TC of NWs bounded with $\{110\}$ surfaces [41] due to the stronger surface-phonon scattering. The atoms on the $\{100\}$ surface possess two dangling bonds, while those on the $\{110\}$ surface possess only one dangling bond. Larger dangling bond density will cause much stronger surface scattering and lead to smaller TC. With the reduction of the main NW cross-section width $L_{w}$, the TC of pristine NWs decreases due to the enhanced surface scattering and confinement effect [40,42-46]. The TC of the pristine NWs obtained in our work is smaller than the values for thin membranes of similar sizes in [11], indicating the importance of boundary scattering. Both screw dislocations and surface resonant structures reduce the TC of NWs by roughly the same amount for every size.

However, it is worth noting that the magnitude of TC reduction can be tuned by Burgers vector length [23,24], resonator dimension, and number density $[8,12,47,48]$. As an illustration, we constructed resonant NWs with different pillar heights $L_{h}$, and the corresponding TCs at $300 \mathrm{~K}$ are calculated. As shown by the inset of Fig. 2, the TC decreases linearly with the increase of pillar height due to the increased number of resonant modes. Normally, when two phonon scattering sources are combined, they do not add their TC reductions if they hinder the modes in a similar frequency range. To check if surface resonators and screw dislocations can produce a synergistic effect on TC reduction, we constructed NWs with both screw dislocations and surface resonators introduced, i.e., SDRe NWs. As can be seen in Fig. 2, the TCs of SDRe NWs are further reduced with a relatively large magnitude compared to the ones of both the SD and Re NWs. The significant reduction of TC in SDRe NWs indicates that phonon resonances can complement dislocations in TC engineering, which is very promising for ultralow TC design.

To clarify the origin of this synergistic effect of surface resonators and screw dislocations, it is essential to study how resonances and dislocations reduce the TC of NWs separately, especially identifying which phonon frequency range that is affected by either. Since according to the kinetic theory [49] the TC is directly related to the phonon group velocities and relaxation times, we calculated the group velocities and relaxation times of the four types of NWs with $L_{w}=4.34 \mathrm{~nm}$ based on lattice dynamics [50] and spectral energy decomposition (SED) [51,52] methods, respectively. In the lattice dynamics calculations, the unit cell length in the periodic direction for the pristine and SD NWs has the same length as the conventional Si lattice parameter $(5.43 \AA)$ while six times the conventional lattice is used for Re and SDRe NWs due to the existence of pillars, which leads to a six times smaller reciprocal lattice compared to that of pristine and SD NWs. In SED calculations, four periodic unit cells are used for velocity calculations, allowing for the calculation of SED on four wave vectors [51,52]. Figure 3(a) shows the average group velocity as a function of frequency. In resonant structures, since the resonant modes themselves possess zero group velocity, it should be excluded for the average group velocity calculations. In this aim, we defined the mode weight factor $[53,54]$ of the pillars $W_{\lambda}=\Sigma_{i \in \text { pillar }} e_{i \alpha}^{\lambda}\left(e_{i \alpha}^{\lambda}\right)^{*}$, with $e_{i \alpha}^{\lambda}$ being the eigenvector component of atom $i$ in direction $\alpha$, and the superscript $*$ denotes conjugation. The summation is over the atoms in pillars and $W_{\lambda}=1$ if the summation is over all the atoms in the unit cell. For the pure resonant modes, which are localized on pillars, $W_{\lambda}$ of the pillar region is close to 1 . In average group velocity calculations, we exclude those modes with a mode weight factor of the pillars region larger than 0.8 , i.e., $W_{\lambda}^{\text {pillar }}>0.8$. With this procedure, we can 

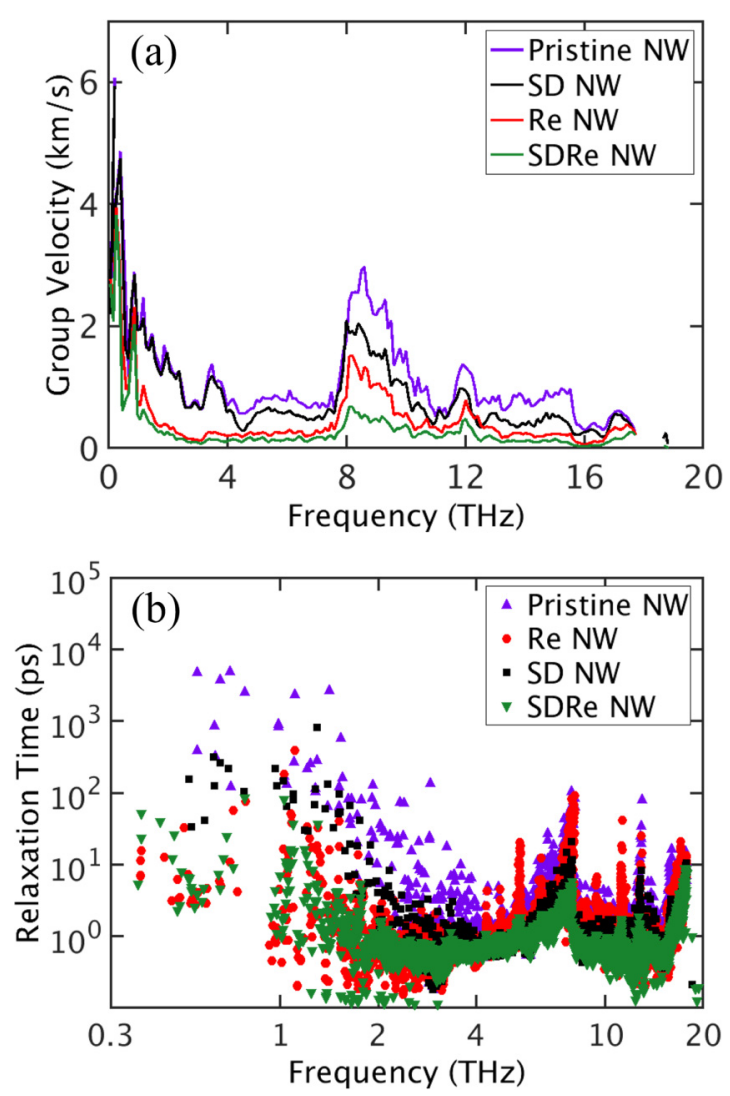

FIG. 3. The averaged group velocity (a) and relaxation time (b) of the pristine, SD, Re, and SDRe NWs, where the NW crosssection width is $4.34 \mathrm{~nm}$ and the height of pillars is $2.17 \mathrm{~nm}$.

effectively exclude the pure resonant modes. From Fig. 3(a) we can find that the inclusion of screw dislocations in NWs does not affect significantly the group velocities at frequencies below $8 \mathrm{THz}$, either for pristine NWs or for NWs with surface resonators. Above $8 \mathrm{THz}$, group velocities are reduced slightly by the dislocation in both cases, but the contribution of these high-frequency modes to thermal transport is less important. Unchanged group velocities indicate that the dispersion relation is almost unaffected by dislocations, revealing the same harmonic vibrational properties. As a result, the TC reduction by dislocation has to originate from the variation of phonon anharmonicity, which determines the relaxation time $\tau$. Contrary to dislocations, surface resonances can greatly reduce the group velocity in NWs in the entire frequency range, regardless of whether dislocations are involved or not. The reduction of group velocities by surface resonators is a result of the hybridization effect between resonant and propagating modes.

Whereas the decrease of the group velocities in resonant structures is responsible for the TC reduction, we found that the MFP of low-frequency modes can be reduced by two orders of magnitude by resonances [8], which is much larger than the observed group velocity reduction. As a consequence, we argue that the relaxation time should also play an important role in modulating phonon transport. Moreover, in a previous work, some of us argued that a screw dislocation cannot directly scatter phonons, but it would hinder phonon transport by enhancing lattice anharmonicity by inducing stress [24]. To evaluate the change of relaxation time by surface resonators and dislocations, we perform SED calculations and extract the relaxation time of each mode for the four NW structures [Fig. 3(b)]. For pristine structures, the relaxation time decreases rapidly with the increase of frequency, and the value for most of the modes beyond $4 \mathrm{THz}$ is in the order of picoseconds. With the inclusion of a screw dislocation in the pristine $\mathrm{NW}$, the relaxation times at all frequencies are reduced, including those of low-frequency acoustic modes. The low-frequency relaxation times are reduced by one order of magnitude below $4 \mathrm{THz}$, beyond which the reduction is much weaker.

With the addition of surface pillars, the relaxation time is slightly decreased above $4 \mathrm{THz}$ compared to the value of pristine structures. However, it is reduced by more than two orders of magnitude below $4 \mathrm{THz}$, which is much stronger than the reduction retreated by dislocations. The strong reduction of relaxation time at low frequencies by resonances indicates that it plays an important role in TC engineering for resonant structures. This finding complements the previous assumptions that resonances mainly reduce the phonon group velocities, highlighting the effect of resonances on relaxation times and its importance for TC reduction. The reduction of relaxation time by flat bands has also been observed in filled skutterudite $[15,16]$. The above analysis indicates that the effect of a screw dislocation on phonon relaxation time is stronger at high frequencies, while that of surface resonances is stronger at low frequencies. As a result, the two phonon manipulation methods can complement each other, and they are able to produce a synergistic effect on relaxation time engineering, which is evidenced by the significant reduction of relaxation time in the whole frequency range in SDRe NWs.

According to the kinetic theory, the TC of each mode can be calculated with the obtained group velocity $v_{g}$ and relaxation time $\tau$ according to $\kappa=c_{v} v_{g}^{2} \tau$, where the classical mode heat capacity $c_{v}=k_{B}$ is used since the TC obtained by MD simulations is classical. Figure 4 shows the TC of each mode in the pristine, Re, SD, and SDRe NWs. To represent the data clearly, we plotted the data in two figures with the SDRe one in Fig. 4(b). The TC of the pristine NW below $4 \mathrm{THz}$ is relatively large, and some of the modes even possess TC larger than $1 \mathrm{~W} \mathrm{~m}^{-1} \mathrm{~K}^{-1}$ due to the long MFP. With the introduction of $\mathrm{SD}$, the TC of the low-frequency modes can be reduced by approximately one order of magnitude, which is in accordance with the relaxation time reduction. At high frequencies, the TC spectral components spread over a broad range, and some of the them are reduced noticeably while the rest of them are almost unchanged. As for Re NWs, the TC spectral components at all frequencies are distributed in a broad range. There are many components in Re NWs possessing very small TC due to the almost zero group velocity. Compared to the SD NWs, the TC of the Re NWs is much smaller below $4 \mathrm{THz}$, which indicates the strong hindrance of low-frequency thermal transport by resonances. For the SDRe NWs [Fig. 4(b)], the mode TC in the entire frequency range is dramatically reduced compared to the value of the pristine structures, which clearly demonstrates the synergistic effect of dislocation and resonances. 

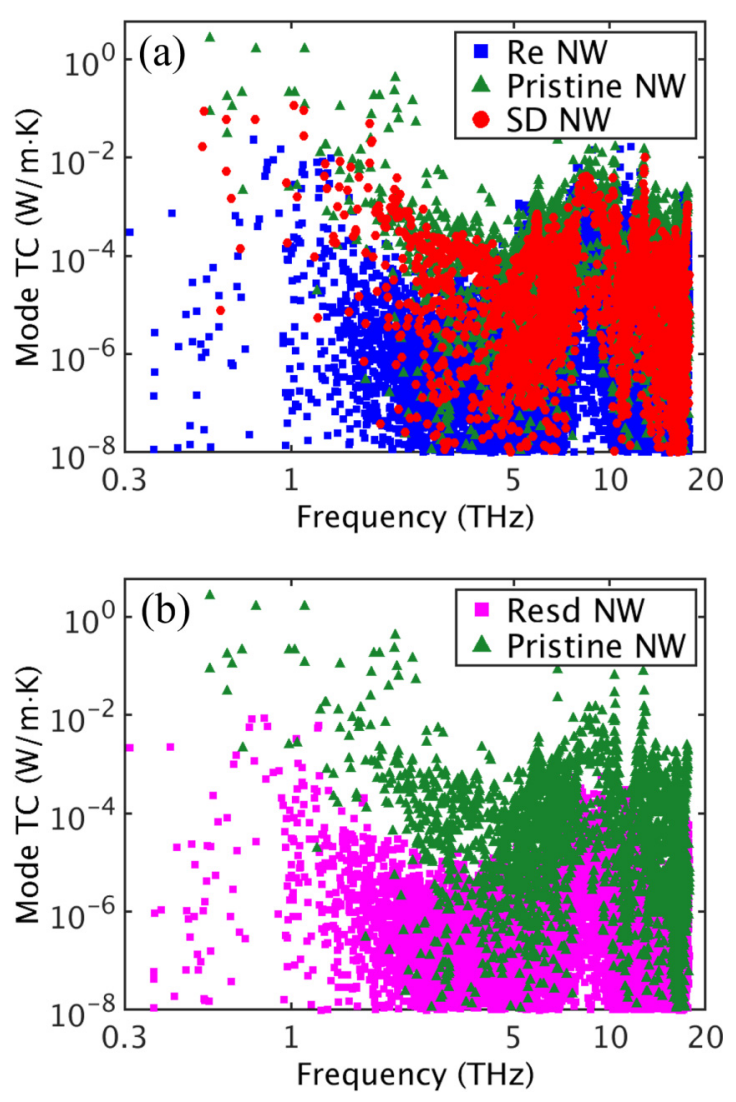

FIG. 4. The thermal conductivity of each mode for the pristine, Re, SD, and SDRe NWs. For a better presentation, the values of pristine, Re, and SD NWs are plotted in (a), while the data for the pristine and SDRe NWs are plotted in (b).

To analyze the variation of relaxation time, we refer to the Fermi Golden Rule. According to the Fermi Golden Rule, the three phonon-phonon scattering rate can be obtained as follows [55]:

$$
\Omega_{ \pm}=\frac{\hbar \pi}{4 N_{0}}\left\{\begin{array}{c}
n_{\lambda^{\prime}}^{0}-n_{\lambda^{\prime \prime}}^{0} \\
n_{\lambda^{\prime}}^{0}+n_{\lambda^{\prime \prime}}^{0}+1
\end{array}\right\}\left|V_{ \pm}^{(3)}\right|^{2} \frac{\delta\left(\omega_{\lambda} \pm \omega_{\lambda^{\prime}}-\omega_{\lambda^{\prime \prime}}\right)}{\omega_{\lambda} \omega_{\lambda^{\prime}} \omega_{\lambda^{\prime \prime}}},
$$

where $\omega_{\lambda}$ is the frequency of the $\lambda$ th modes, and $V_{ \pm}^{(3)}$ represents the scattering matrix element, which can be cast as

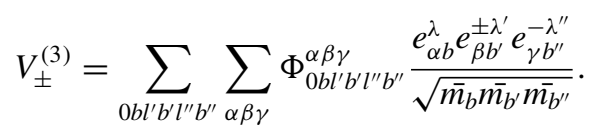

Here $\Phi$ denotes the third-order force constant, and $e_{\alpha b}^{\lambda}$ is the eigenvector component of atom $b$ in direction $\alpha$ of the $\lambda$ th mode. $\bar{m}$ and $l$ are the average atom mass and unit cell index, respectively. As illustrated by Eq. (1), there are two quantities that affect the relaxation time (inverse of scattering rate), i.e., the scattering channels and the scattering matrix elements. The former is characterized by the phase space, while the latter is determined by the anharmonic force constants as well as the spatial overlap of the corresponding modes [Eq. (2)]. To clarify which factor is responsible for the relaxation time reduction by the dislocations and the resonators, we calculated the phase space of three-phonon scattering for each mode,
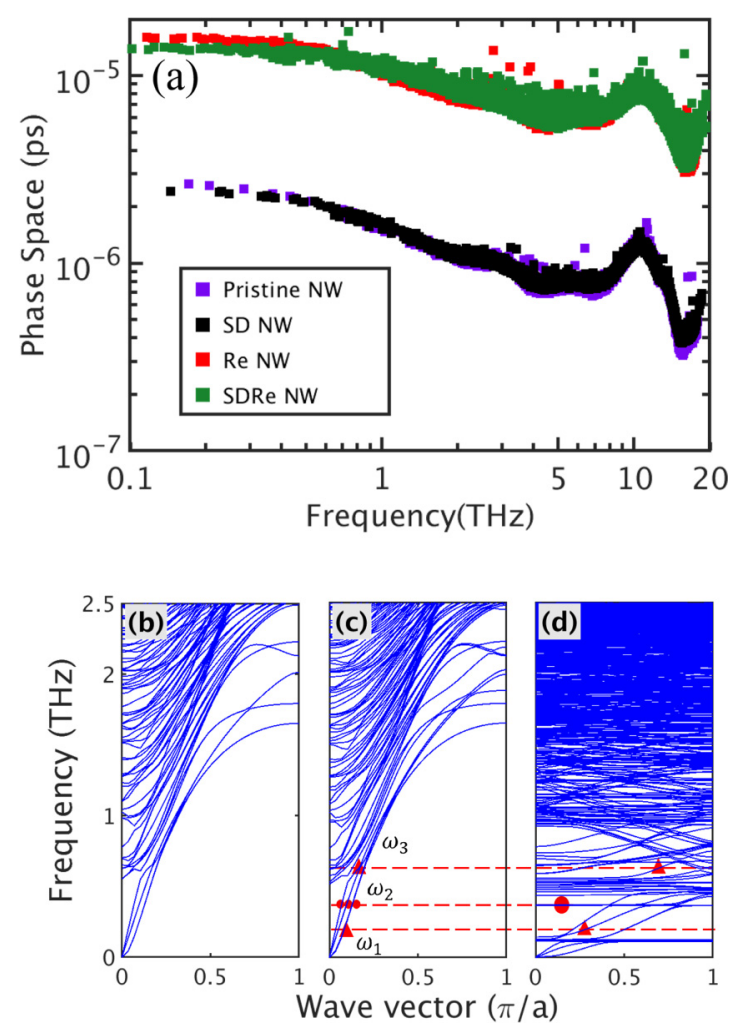

FIG. 5. (a) The phase space of three-phonon scattering for the pristine, SD, Re, and SDRe NWs; phonon dispersion curves of the pristine NW (b), SD NW (c), and Re NW (d). The cross-section width of all NWs is $4.34 \mathrm{~nm}$ and the height of the pillars is $2.17 \mathrm{~nm}$. The lattice parameter $a=5.43 \AA$ in (b) and (c), and $a=32.6 \AA$ in (d).

which is defined as $[16,56,57]$

$$
P_{3}\left(\omega_{\lambda}\right)=\frac{1}{N_{k}^{2} N_{b}^{3}} \sum_{\lambda^{\prime}, \lambda^{\prime \prime}} \delta\left[\omega_{\lambda} \pm \omega_{\lambda^{\prime}}-\omega_{\lambda^{\prime \prime}}\right],
$$

where $N_{k}$ and $N_{b}$ are the numbers of $k$ points and phonon branches, respectively. The phase space is known to have a strong negative correlation with TC [56], and its value is between 0 and 1 . The actual values are normally several orders of magnitude smaller than 1. Figure 5(a) illustrates the calculated phase space of the four structures calculated according to Eq. (3). In general, the phase space of all four structures decreases with frequency with a small peak around $10 \mathrm{THz}$. As reported in the figure, the phase space of SD NW is almost identical to that of the pristine structure. This is a consequence of the fact that dislocations do not alter significantly phonon dispersion relations, except for breaking the degeneracy of some branches, e.g., the flexural acoustic branch [Fig. 5(c)]. The unaltered phase space reveals that the relaxation time reduction, originated from dislocations, arises from the increased absolute value of the scattering matrix elements $V_{ \pm}^{(3)}$, which indicates that bonds become more anharmonic. The similarity observed in the dispersion relations of dislocated and pristine NWs reveals that the harmonic properties are almost not affected by the dislocation, indicating the same spatial overlap between different modes. As a result, the reduced values of the scattering matrix ele- 
ments should come from the enhancement of phonon-phonon coupling constants. The unaltered harmonic properties might be due to the fact that the bond length variations with the inclusion of a dislocation are small. Consequently, the second derivative of potential energy, which arises mostly from the two-body interactions in the Stillinger-Weber potential, will not change. However, the shear displacement induced by the SD can change the bond angles more significantly than the bond length, which finally causes the change in anharmonicity.

Different from dislocations, the phase space for phonon scattering of Re NWs is largely enhanced at all frequencies compared to that of the pristine structure, indicating that the scattering channels are greatly increased by resonators. In fact, when looking at the phonon dispersion relations, the flat resonant bands crossing the entire Brillouin zone can make the momentum and energy conservation much more probable during the scattering process. For example, taking three phonon frequencies with the energy conservation rule $\omega_{1}+\omega_{2}=\omega_{3}$ (absorption process), where $\omega_{2}$ refers to the resonant frequency, it can always satisfy momentum conservation by adjusting the wave vector of the second mode since the resonant mode dispersion relation is flat [Fig. 5(d)]. In contrast, in pristine or dislocated structures the possibility of finding a mode to satisfy energy and momentum conservation is much lower, as there are only a few discrete modes at the three frequencies [Fig. 5(c)]. The results also show that the relaxation time at high frequencies is only slightly reduced by the presence of resonators, as illustrated in Fig. 3(b), while the phase space is enhanced dramatically at all frequencies. This phenomenon is attributed to the spatial separation between resonant and propagating modes. As analyzed above, to enable the scattering, the participating modes need to spatially overlap. However, at high frequencies the modes are relatively localized compared to the low-frequency ones. Since the resonant modes are mostly localized on pillars, it is difficult for the propagating optical modes to couple with the resonant ones. As a result, most of the scattering matrix elements are essentially zero for the channels related to the resonant modes, while the spatial distribution of low-frequency acoustic modes normally extends to all atoms with pillars included [8]. Thus they can always overlap with resonant modes. Consequently, the resonances have a significant impact on the relaxation time of low-frequency modes as almost all the increased scattering channels can contribute to an effective scattering. In summary, our analysis shows that in resonant NWs, the high-frequency phonons are mostly modified via group velocity reduction, while the low-frequency modes are engineered via relaxation time reduction.

The distribution of the resonant modes could be estimated by considering the isolated pillars (unconnected to the main structure) as indicated in Ref. [11] by Honarvar et al. It has been demonstrated that pillars with a larger cross-section possess wider distributions of resonant modes than taller pillars of the same volume. On the other hand, the resonant modes produced by the pillars are similar to the well-known closed organ-pipe modes, the frequency of which can be estimated as $\frac{(2 n+1) v_{g}}{4 L}$, where $n$ and $v_{g}$ are an integer and the group velocity of the mode forming the corresponding resonances, respectively. $L$ is the length confining the resonant modes. For the first resonant frequency, the two pillars on the opposite sides contribute to the vibrations [8], thus $L=2 L_{h}+L w=8.6 \mathrm{~nm}$ in this case is the height of the two pillars plus the side length of the main NW. The resonant coupling for the first flat band occurs between longitudinal modes. By using lattice dynamics, we calculated the longitudinal phonon group velocity in a $\mathrm{NW}$ with a cross-section of $1.09 \times 1.09 \mathrm{~nm}^{2}$ as $4.6 \mathrm{~km} / \mathrm{s}$. As a result, the estimated resonant frequency is around $0.13 \mathrm{THz}$, which is close to the observed resonant frequency of $0.12 \mathrm{THz}$.

In general, defects scatter phonons directly and can effectively impede the high-frequency (e.g., above $2 \mathrm{THz}$ for alloying) phonon transport by shortening their relaxation times. Due to the longer wavelengths of low-frequency phonons, they get less scattered by lattice defects. In contrast with other scattering sources, our previous work demonstrated that dislocations do not scatter phonons directly; instead, they hinder phonon transport by increasing the lattice anharmonicity [24]. However, it is still unclear how strongly the anharmonicity is enhanced at all frequencies. Here, as illustrated by the obtained relaxation time, we find that the helical dislocation reduces relaxation time not only at high frequencies, but also at low frequencies, which is the advantage of dislocations over other scattering sources in manipulating phonon transport. At frequencies below $4 \mathrm{THz}$, the relaxation time can be reduced approximately by one order of magnitude. We ascribe this phenomenon to the nonhomogeneous stress field produced by screw dislocations. It is well known that screw dislocations can produce strong shear stress [58], and in the core region the atoms even suffer plastic deformation. To check the distribution of stress in SD NWs, we calculated the lateral atomic shear stress profile at $300 \mathrm{~K}$ with $L_{w}=4.34 \mathrm{~nm}$ according to the equation

$$
\sigma=\sqrt{\sigma_{x z}^{2}+\sigma_{y z}^{2}}
$$

where $\sigma_{x z}$ and $\sigma_{y z}$ are the shear stress along the $z$ direction in the $x z$ and $y z$ plane, respectively. They are components of the atomic stress tensor [59]:

$$
\boldsymbol{\sigma}=\frac{1}{V_{i}}\left\{-m_{i} \boldsymbol{v}_{i} \otimes \boldsymbol{v}_{i}+\frac{1}{2} \sum_{j} \boldsymbol{r}_{i j} \otimes \boldsymbol{f}_{i j}\right\},
$$

where $V_{i}, m_{i}$, and $\boldsymbol{v}_{i}$ are the volume, mass, and velocity of atom $i$, respectively. $\boldsymbol{r}_{i j}$ and $\boldsymbol{f}_{i j}$ are the position vector and force between atoms $i$ and $j$. The corresponding results are depicted in Fig. 6(a). All the illustrated stress profiles are averaged over $1 \mathrm{~ns}$ with one calculation every $10 \mathrm{fs}$. The stress profile produced by SD reveals an azimuthal invariance and a maximum value at the circle defined by a critical radius $r_{c}$, below which the stress is small due to the plastic deformation. The shear stress decreases with the increase of radius above $r_{c}$. We can also observe that the surface stress exists due to dangling bonds. To have a better idea of the radius-dependent stress distributions, we plot the shear stress as a function of radius in Fig. 6(b). According to this latter figure, the radius corresponding to the maximum stress is about $r_{c}=0.54 \mathrm{~nm}$ (the dashed vertical line), which is very close to the magnitude of the Burgers vector. As a result, the plastic deformation area caused by a SD has a radius set by the Burgers vector. This 

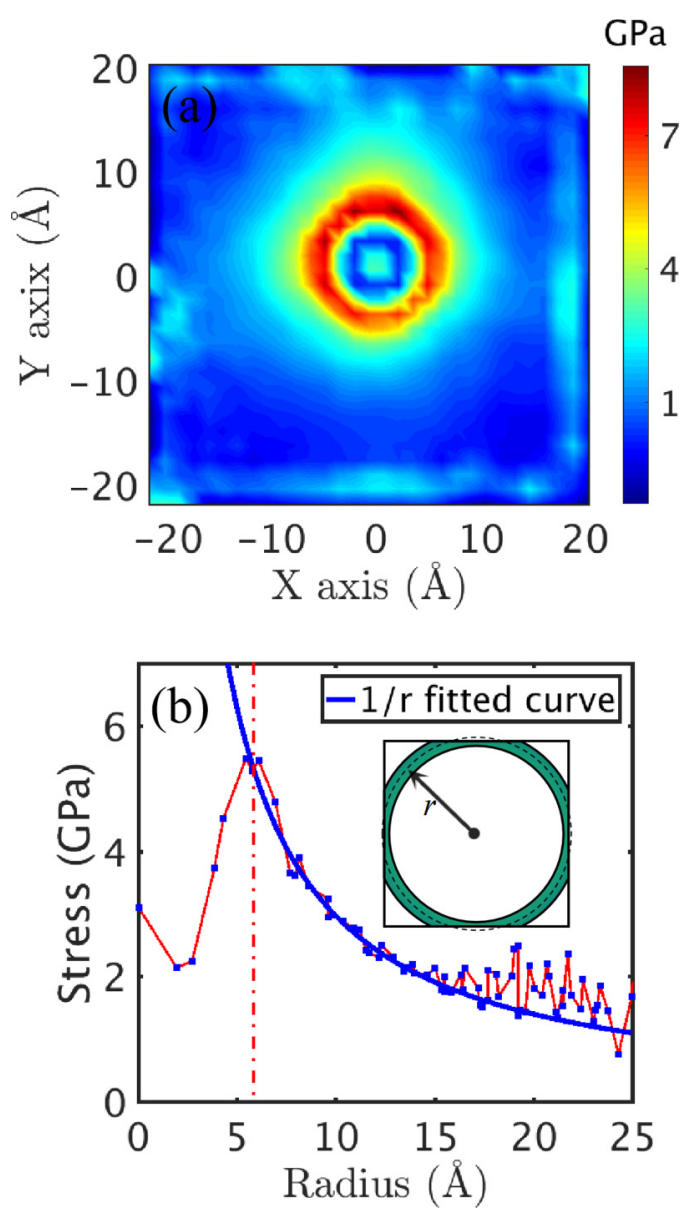

FIG. 6. (a) The lateral shear stress profile and (b) the radiusdependent stress for SD NW with $L_{w}=4.34 \mathrm{~nm}$. The inset of (b) illustrates a schematized shell for stress calculation corresponding to radius $r$.

statement is also valid for larger Burgers vectors, as shown in Appendix B. Beyond $r_{c}$, the stress decreases with an increase in the radius, and it can be well fitted by an $r^{-1}$ dependence, which is in good accordance with the dislocation theory [58].
The stress oscillations at a larger radius are due to the influence of surface stress. In our calculations, the stress of each radius is averaged over all atoms in the corresponding shell, as illustrated by the inset of Fig. 6(b). Since the surface atoms contain dangling bonds, the velocity and position variations of these atoms are larger than those of inner atoms, which eventually lead to the stress fluctuation at a large radius.

To better understand the effect of nonhomogeneous stress on phonon transport in SD NWs, we also perform the corresponding radius decomposition on the phonon density of states (DOS) [60] for pristine and SD NWs. As shown in Fig. 7(a), the DOS is identical for the shells without any surface atoms $(r \leqslant 1.63 \mathrm{~nm})$ in pristine NWs, while the surface atoms will induce the shift of DOS peaks to lower frequencies, corresponding to the softening of phonons. In a SD NW, the peaks of DOS for atoms located in the NW core regions are slightly shifted toward lower frequencies with a more pronounced effect at high frequencies, which means that SD can lead to a slight phonon softening [61]. However, this effect is relatively small compared to that induced by surface atoms. The weak radius-dependent DOS (except the surface region) in the SD NW indicates that the vibrational frequencies will not be altered by SD, which is in agreement with our dispersion and group velocity analysis.

The increase in temperature can enhance the scattering of phonons by increasing the anharmonicity. To check how temperature can affect the thermal transport in our resonant and screw dislocated systems, we perform TC calculations at different temperatures as reported in Fig. 8. Note that we did not present the TC of NWs with resonant structures at 550 and $600 \mathrm{~K}$ as the thin pillars are unstable at such high temperatures. In the current study, the cross-section of the pillars is only $1.09 \times 1.09 \mathrm{~nm}^{2}$ and the surface-to-volume ratio is too large $\left(2.5 \mathrm{~nm}^{-1}\right)$. As a result, the large surface energy due to the dangling bonds makes the pillars melt at lower temperatures $[62,63]$. However, when the cross-section is increased to $1.63 \times 1.63 \mathrm{~nm}^{2}$, the pillars become stable at $600 \mathrm{~K}$. When temperature increases, the TC of all structures decreases in general except those of SD NWs at 550 and $600 \mathrm{~K}$, at which the TC is increased compared to that at lower temperatures. This abnormal phenomenon arises from
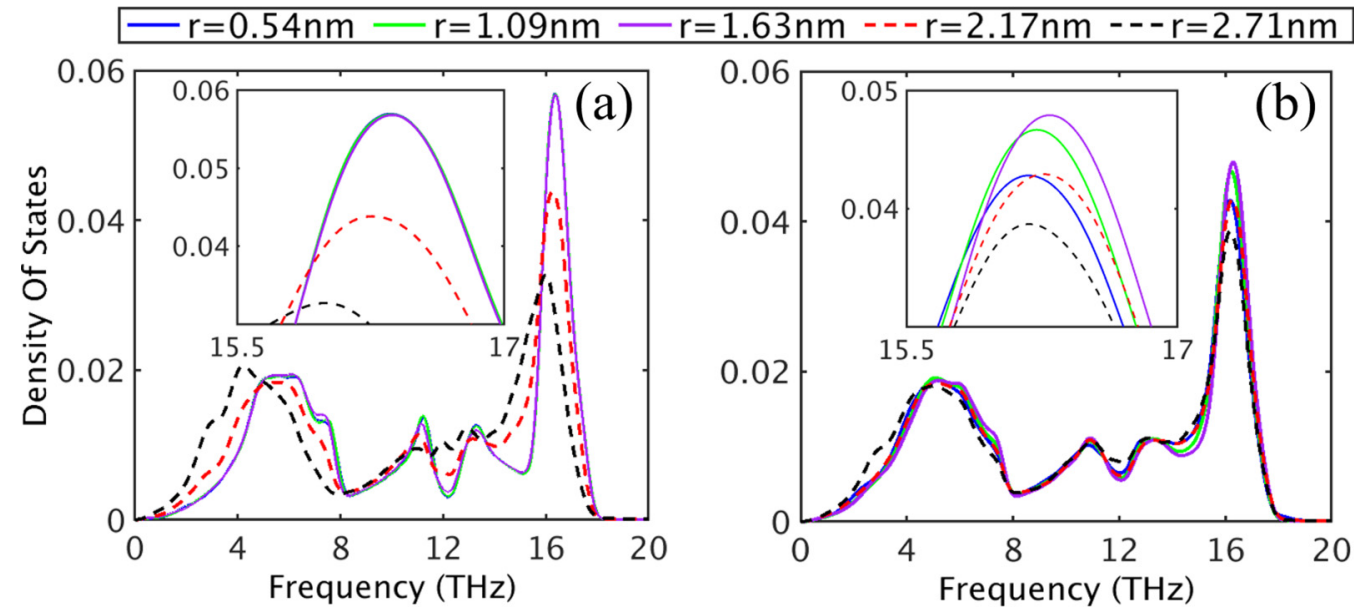

FIG. 7. Radial decomposition of DOS for pristine (a) and SD (b) NWs with the cross-section width $L_{w}=4.34 \mathrm{~nm}$. The insets are the corresponding zoom in the plot for the frequency range between 15.5 and $17 \mathrm{THz}$. 


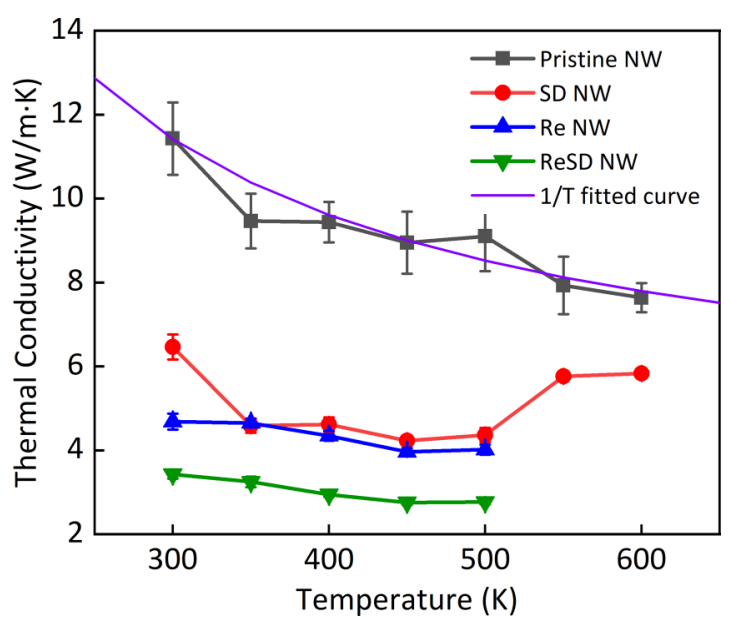

FIG. 8. Temperature-dependent thermal conductivity for pristine NWs, SD NWs, Re NWs, and SDRe NWs with the NW cross-section width $L_{w}=3.26 \mathrm{~nm}$. At temperature higher than $500 \mathrm{~K}$, the small surface resonator structures are unstable, hence the corresponding thermal conductivities are not presented.

the dislocation recovery at high temperatures, which will be explained later. For the pristine NW, the TC can be well fitted by the $1 / T$ relation, a signature of anharmonic scattering. For many scattering resources such as alloying, the resulting TC is usually temperature-independent due to the strong scattering of high-frequency phonons by defects $[8,64-66]$, which makes the anharmonic effect negligible even at high temperatures since the temperature-induced anharmonicity is only noticeable at high frequencies. However, for the screw dislocated and resonant structures, we find that the TC can still be decreased with the elevation of temperature, meaning that the hindrance of high-frequency phonons by dislocation and resonance is not as strong as that generated by temperature. At $500 \mathrm{~K}$, the TC can be reduced by a factor of 2 induced by dislocations or resonances, which reveals the ability to tune the TC at high temperature by the two sources. The combination of screw dislocations and resonators can further decrease the TC of NWs at the studied temperatures, indicating that the synergistic effect can be preserved at all temperatures. Even for the SDRe structure, we can still observe the reduction of TC with the increase of temperature, which means that the high-frequency phonons still can contribute to thermal transport at low temperature.

At 550 and $600 \mathrm{~K}$, we note that there is an abnormal TC increase for SD NWs. After a careful check of all conditions, we find that these outliers are due to the partial recovery of dislocations at these two temperatures. To have a clear idea of the dislocation recovery, we plot the shear stress profile for SD NWs at 500 and $550 \mathrm{~K}$ in Fig. 9. It can be clearly observed that at $500 \mathrm{~K}$, stress induced by screw dislocation is large. The critical radius $r_{c}$ for the core region with plastic deformation is still around the value of the Burgers vector. Beyond $r_{c}$ the stress profile can be fitted by a $1 / r^{0.9}$ relation. However, when the temperature rises to $550 \mathrm{~K}$, the dislocation-induced stress completely disappears; only surface stress can be observed in Fig. 9(c). In the middle region, the stress is almost the same at different radii until surface atoms are involved, which
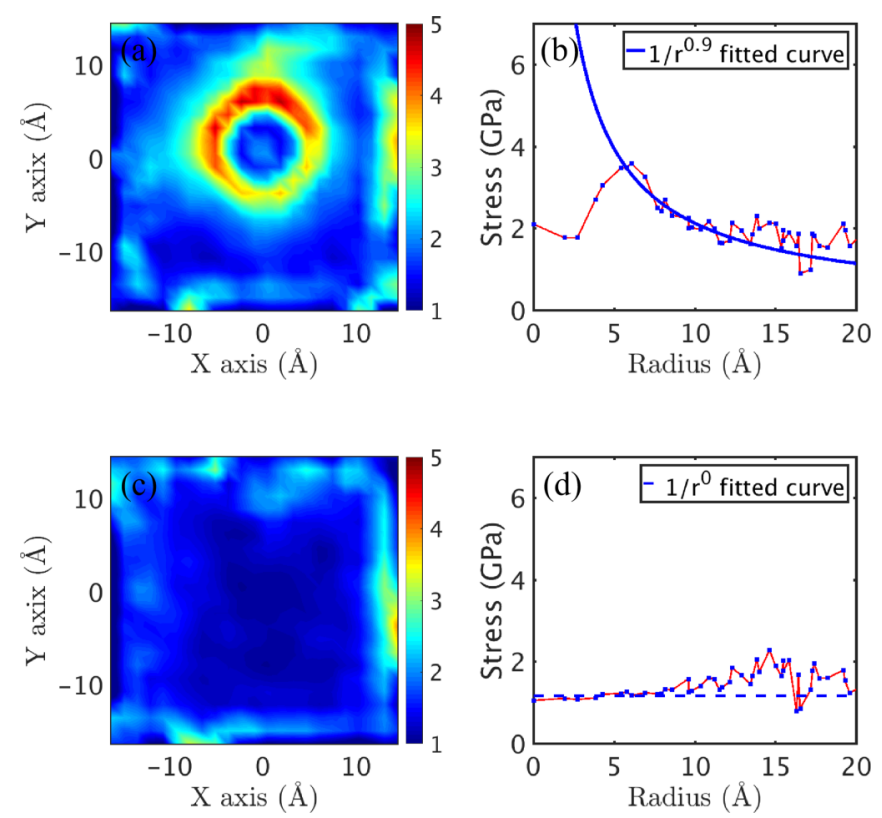

FIG. 9. The lateral shear stress profile and the radius-dependent stress for SD NW with $L_{w}=3.26 \mathrm{~nm}$ at $500 \mathrm{~K}$ (a),(b) and $550 \mathrm{~K}$ (c),(d).

reveals the recovery from the screw dislocation and leads to the abnormal increase of TC with temperature. However, we find that the TC did not recover back to the value of pristine structures, which might be due to the incomplete recovery from the dislocation. We have tried to relax the structures for 10 further nanoseconds and find that the TC is the same. So we think this incomplete recovery is an intrinsic property of screw dislocations.

\section{CONCLUSIONS}

The thermal transport properties of Si NWs with screw dislocation and surface resonators have been studied systematically by molecular dynamics simulations. We find that both screw dislocations and surface resonators can reduce the TC of NWs noticeably. The screw dislocation has almost no effect on the harmonic properties, and the TC modification is fully achieved by enhancing the anharmonicity of NWs. Differing from other defects such as alloys, which scatter high-frequency phonons only, screw dislocations can reduce the relaxation time in the whole frequency range due to the nonhomogeneous stress field. As for the resonator structure, we uncover that it not only reduces the group velocities of phonons through the resonant hybridization, but it also can diminish the relaxation time strongly at low frequencies, a mechanism unrecognized before. The strong shortening of low-frequency phonon relaxation times is caused by the increased number of scattering channels. As a result, the resonant structures can modulate phonon transport through the reduction of group velocities at high frequencies and the reduction of relaxation time at low frequencies. The strong hindrance of low- and high-frequency phonon transports by resonators and screw dislocations is eventually a very promising combination to engineer phonon transport at all 
frequencies. We also uncover that the dislocations can recover back at temperatures above $550 \mathrm{~K}$, yielding an abnormal TC increase with temperature at $550 \mathrm{~K}$. Our theoretical results not only provide insights into the $\mathrm{TC}$ reduction mechanisms produced by screw dislocations and surface resonators, but they also illustrate a paradigm for ultralow TC engineering through the manipulation of the entire spectrum of phonons contributing to energy transport.

\section{ACKNOWLEDGMENTS}

This work is supported by the National Natural Science Foundation of China (Grant No. 11804242), the China Postdoctoral Science Foundation (Grant No. 2019M651945), Collaborative Innovation Center of Suzhou Nano Science \& Technology (Nano-CIC), the Priority Academic Program Development of Jiangsu Higher Education Institutions (PAPD), the 111 Project, and Joint International Research Laboratory of Carbon-Based Functional Materials and Devices.

\section{APPENDIX A: COMPARISON BETWEEN THE STRESS FIELDS}

As discussed in Sec. III, the SD can generate a nonhomogeneous strain. Here we provide a comparison on stress distributions among the pristine, SD, Re, and SDRe NWs. The cross-section of the main NW considered is $L_{w}=4.34 \mathrm{~nm}$. It can be clearly observed from Fig. 10 that the stress is small in the NW except that there is a surface stress for the pristine NW. For the Re NW, the stress distribution in the main NW is similar to the case of the pristine structure. Due to the relatively small cross-section $\left(1.09 \times 1.09 \mathrm{~nm}^{2}\right)$ of our pillars, the surface atom ratio is large, which makes the stress larger than for the atoms inside the main NW. With the introduction of a $\mathrm{SD}$ in the middle of the NW, a nonhomogeneous stress field
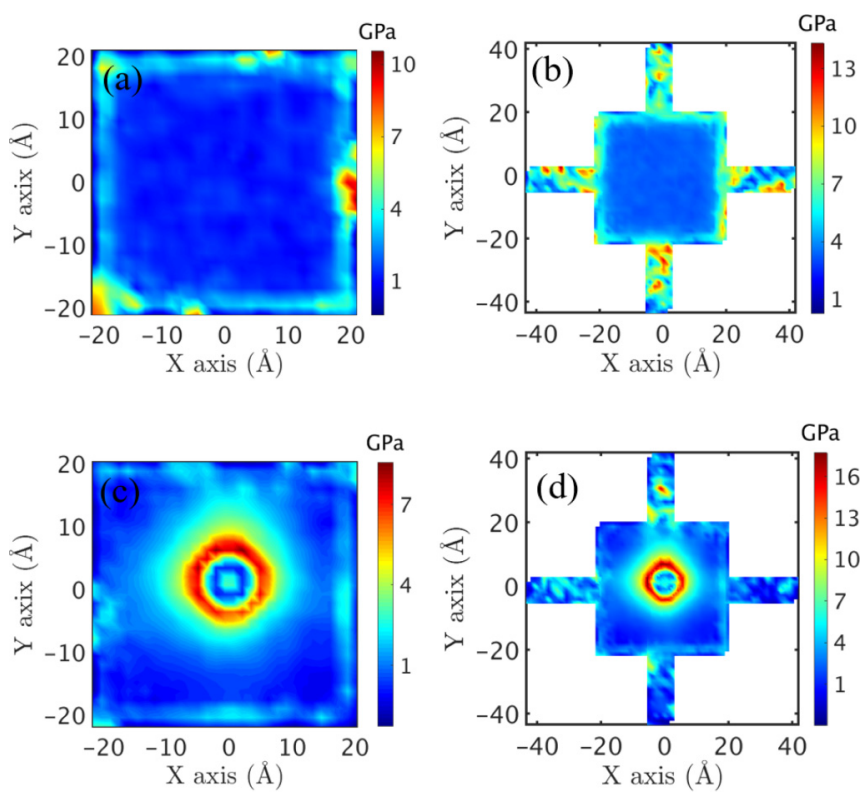

FIG. 10. The lateral shear stress profile of (a) pristine NW, (b) Re $\mathrm{NW}$, (c) SD NW, and (d) SDRe NW, the cross-section width $L_{w}=$ $4.34 \mathrm{~nm}$ is generated in both SD and SDRe NWs. The stress field has rotational symmetry with a maximum value at a critical radius. Moreover, the critical radius for plastic-elastic deformation in both SD and SDRe NWs is the same, corresponding to the size of the Burgers vector, indicating that the resonant structure will not affect the SD induced stress distribution.

\section{APPENDIX B: BURGERS VECTOR AND TEMPERATURE EFFECT ON DISLOCATION INDUCED STRESS}

The nonhomogeneous stress field created by SD should be dependent on the dislocation magnitude, especially for the critical radius of the maximum stress. To check this point, we calculated the radius-dependent stress distributions for SD NWs with different Burgers vectors ranging from $\mathbf{1 b}$ to $\mathbf{3 b}$. Since the largest Burgers vector is $\mathbf{3 b}$, we choose a NW with a larger cross-section $\left(L_{w}=7.6 \mathrm{~nm}\right)$ to avoid the overlap between the surface stress and stress produced by dislocation. The obtained results are demonstrated in Fig. 11(a), which reports that a critical radius corresponding to the maximum stress indeed enlarges with the increase of the Burgers vector. For all the Burgers vectors investigated, the stress within the circle highlighting the critical radius is relatively small. This region corresponds to the plastic deformation region. Beyond the critical radius, the stress reduces with the radius gradually. The stress beyond the critical radius can be well fitted by a $1 / r$ relation for the three Burgers vectors, which is in good accordance with the traditional dislocation theory [58]. It is also interesting to observe that the critical radius between plastic and elastic deformations corresponds very well to the Burgers vector magnitude for all three cases, as shown by the dashed vertical lines in Fig. 11(a). As a result, we can
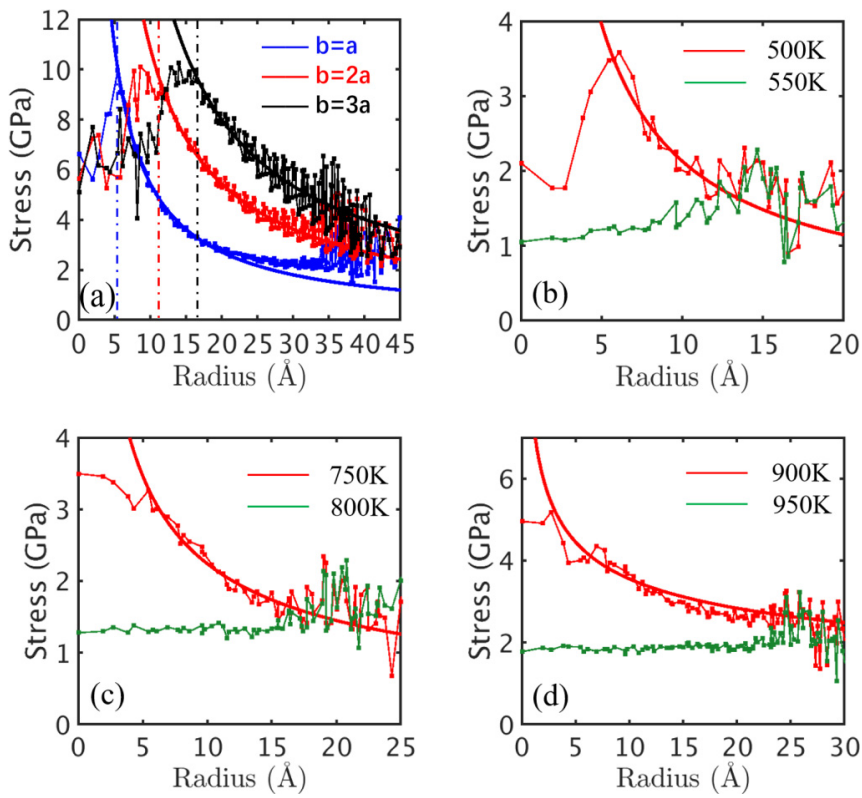

FIG. 11. The radius-dependent stress for the NWs with Burgers vectors of $\mathbf{1 b}, \mathbf{2} \mathbf{b}$, and $\mathbf{3} \mathbf{b}$ (a). The cross-section of the NWs is $7.6 \times 7.6 \mathrm{~nm}^{2}$. The radius-dependent stress of SD NWs with Burgers vector of $\mathbf{1 b}$ at temperatures before and after screw dislocation recovery with different cross-sections: (b) $3.26 \times 3.26 \mathrm{~nm}^{2}$, (c) $4.34 \times 4.34 \mathrm{~nm}^{2}$, and (d) $5.43 \times 5.43 \mathrm{~nm}^{2}$. 
conclude that the plastic deformation area induced by SD is within the radius of the corresponding Burgers vector, beyond which elastic deformation takes place.

In Fig. 9, we showed that the screw dislocation can partially recover at elevated temperatures, which was quantified by the stress field. To have a more detailed study on this SD recovery temperature, we performed the radius-dependent stress calculations for SD NWs with different cross-sections and a 1b Burgers vector. The corresponding results are displayed in Figs. 11(b)-11(d). For all the cross-sections, we can find that there is a SD recovery temperature, below which the stress is large, and the radius-dependent stress follows the dislocation theory. However, at temperatures higher than the SD recovery temperature, the stress is almost independent of radius, indicating that the SD induced stress disappeared. When the cross-section of the NW is $3.26 \times 3.26 \mathrm{~nm}^{2}$, the SD recovery temperature is between 500 and $550 \mathrm{~K}$. This recovery temperature increases to $750-800 \mathrm{~K}$ in the NW with cross-section $4.34 \times 4.34 \mathrm{~nm}^{2}$ and further increases to 900 $950 \mathrm{~K}$ when the cross-section is enlarged to $5.43 \times 5.43 \mathrm{~nm}^{2}$. In general, the SD recovery temperature increases with the enlargement of the NW cross-section.
[1] A. Katre, J. Carrete, B. Dongre, G. K. H. Madsen, and N. Mingo, Phys. Rev. Lett. 119, 075902 (2017).

[2] S. Hu, H. Zhang, S. Xiong, H. Zhang, H. Wang, Y. Chen, S. Volz, and Y. Ni, Phys. Rev. B 100, 075432 (2019).

[3] P. Hyldgaard and G. D. Mahan, Phys. Rev. B 56, 10754 (1997).

[4] P. Martin, Z. Aksamija, E. Pop, and U. Ravaioli, Phys. Rev. Lett. 102, 125503 (2009).

[5] L. D. Hicks and M. S. Dresselhaus, Phys. Rev. B 47, 16631 (1993).

[6] J. Recatala-Gomez, A. Suwardi, I. Nandhakumar, A. Abutaha, and K. Hippalgaonkar, ACS Appl. Energy Mater. 3, 2240 (2020).

[7] B. L. Davis and M. I. Hussein, Phys. Rev. Lett. 112, 055505 (2014).

[8] S. Xiong, K. Sääskilahti, Y. A. Kosevich, H. Han, D. Donadio, and S. Volz, Phys. Rev. Lett. 117, 025503 (2016).

[9] S. Neogi, J. S. Reparaz, L. F. C. Pereira, B. Graczykowski, M. R. Wagner, M. Sledzinska, A. Shchepetov, M. Prunnila, J. Ahopelto, C. M. Sotomayor-Torres, and D. Donadio, ACS Nano 9, 3820 (2015).

[10] X.-K. Chen, J. Liu, Z.-X. Xie, Y. Zhang, Y.-X. Deng, and K.-Q. Chen, Appl. Phys. Lett. 113, 121906 (2018).

[11] H. Honarvar and M. I. Hussein, Phys. Rev. B 97, 195413 (2018).

[12] H. Zhang, B. Sun, S. Hu, H. Wang, Y. Cheng, S. Xiong, S. Volz, and Y. Ni, Phys. Rev. B 101, 205418 (2020).

[13] A. Giri and P. E. Hopkins, Phys. Rev. B 98, 045421 (2018).

[14] X. Huang, D. Ohori, R. Yanagisawa, R. Anufriev, S. Samukawa, and M. Nomura, ACS Appl. Mater. Interfaces 12, 25478 (2020).

[15] W. Li, J. Carrete, G. K. H. Madsen, and N. Mingo, Phys. Rev. B 93, 205203 (2016).

[16] W. Li and N. Mingo, Phys. Rev. B 91, 144304 (2015).

[17] T. Tadano, Y. Gohda, and S. Tsuneyuki, Phys. Rev. Lett. 114, 095501 (2015).

[18] Z. Zhang, S. Hu, T. Nakayama, J. Chen, and B. Li, Carbon 139, 289 (2018).

[19] S. Neogi and D. Donadio, Phys. Rev. Appl. 14, 024004 (2020).

[20] H. Honarvar and M. I. Hussein, Phys. Rev. B 93, 081412(R) (2016).

[21] S. Xiong, D. Selli, S. Neogi, and D. Donadio, Phys. Rev. B 95, 180301(R) (2017).

[22] M. J. Bierman, Y. K. A. Lau, A. V. Kvit, A. L. Schmitt, and S. Jin, Science 320, 1060 (2008).

[23] Y. Ni, S. Xiong, S. Volz, and T. Dumitrica, Phys. Rev. Lett. 113, 124301 (2014).
[24] S. Xiong, J. Ma, S. Volz, and T. Dumitrica, Small 10, 1756 (2014).

[25] S. A. Morin and S. Jin, Nano Lett. 10, 3459 (2010).

[26] K. A.Dick, K. Deppert, M. W. Larsson, T. Mårtensson, W. Seifert, L. R. Wallenberg, and L. Samuelson, Nat. Mater. 3, 380 (2004).

[27] A. Dong, R. Tang, and W. E. Buhro, J. Am. Chem. Soc. 129, 12254 (2007).

[28] K. Jun and J. M. Jacobson, Nano Lett. 10, 2777 (2010).

[29] S. Plimpton, J. Comput. Phys. 117, 1 (1995).

[30] F. H. Stillinger and T. A. Weber, Phys. Rev. B 31, 5262 (1985).

[31] J. Chen, G. Zhang, and B. Li, Nano Lett. 10, 3978 (2010).

[32] Z. Rashid, L. Zhu and W. Li, Phys. Rev. B 97, 075441 (2018).

[33] W. G. Hoover, Phys. Rev. A 31, 1695 (1985).

[34] G. J. Martyna, D. J. Tobias, and M. L. Klein, J. Chem. Phys. 101, 4177 (1994).

[35] M. S. Green, J. Chem. Phys. 22, 398 (1954).

[36] R. Kubo, J. Phys. Soc. Jpn. 12, 570 (1957).

[37] R. Kubo, M. Toda, and N. Hashitsume, Statistical Physics II, Springer Series in Solid-State Sciences (Springer, Berlin, 1981).

[38] J. R. Lukes and H. Zhong, J. Heat Transf. 129, 705 (2007).

[39] J. E. Turney, A. J. H. McGaughey, and C. H. Amon, Phys. Rev. B 79, 224305 (2009).

[40] I. Ponomareva, D. Srivastava, and M. Menon, Nano Lett. 7, 1155 (2007).

[41] Y. Zhou, X. Zhang, and M. Hu, Nano Lett. 17, 1269 (2017).

[42] D. Donadio and G. Galli, Phys. Rev. Lett. 102, 195901 (2009).

[43] S. G. Walkauskas, D. A. Broido, K. Kempa, and T. L. Reinecke, J. Appl. Phys. 85, 2579 (1999).

[44] J. Mao, Z. Liu, and Z. Ren, npj Quantum Mater. 1, 16028 (2016).

[45] A. Soleimani, H. Araghi, Z. Zabihi, and A. Alibakhshi, Comput. Mater. Sci. 142, 346 (2018).

[46] D. Ma, H. Ding, H. Meng, L. Feng, Y. Wu, J. Shiomi, and N. Yang, Phys. Rev. B 94, 165434 (2016).

[47] M. I. Hussein, C.-N. Tsai, and H. Honarvar, Adv. Funct. Mater. 30, 1906718 (2020).

[48] H. Honarvar, L. Yang, and M. I. Hussein, Appl. Phys. Lett. 108, 263101 (2016).

[49] J. Ziman, Electrons and Phonons: The Theory of Transport Phenomena in Solids (Clarendon, Oxford, 1960).

[50] M. Born and K. Huang, Dynamical Theory of Crystal Lattices (Clarendon, Oxford, 1998).

[51] A. S. Henry and G. Chen, J. Comput. Theor. Nanosci. 5, 141 (2008) 
[52] J. A. Thomas, J. E. Turney, R. M. Iutzi, C. H. Amon, and A. J. H. McGaughey, Phys. Rev. B 81, 081411(R) (2010).

[53] M. Hu, X. Zhang, K. P. Giapis, and D. Poulikakos, Phys. Rev. B 84, 085442 (2011).

[54] J. L. Feldman and N. Bernstein, Phys. Rev. B 70, 235214 (2004).

[55] J. Fabian and P. B. Allen, Phys. Rev. Lett. 77, 3839 (1996).

[56] L. Lindsay and D. A. Broido, J. Phys.: Condens. Matter 20, 165209 (2008).

[57] W. Li, J. Carrete, N. A. Katcho, and N. Mingo, Comput. Phys. Commun. 185, 1747 (2014).

[58] P. M. Anderson, J. P. Hirth, and J. Lothe, Theory of Dislocations (Cambridge University Press, Cambridge, 2017).

[59] A. P. Thompson, S. J. Plimpton, and W. Mattson, J. Chem. Phys. 131, 154107 (2009).
[60] F. Sansoz, Nano Lett. 11, 5378 (2011).

[61] V. Taupin, S. Varadhan, J. Chevy, C. Fressengeas, A. J. Beaudoin, M. Montagnat, and P. Duval, Phys. Rev. Lett. 99, 155507 (2007).

[62] S. Xiong, W. Qi, Y. Cheng, B. Huang, M. Wang, and Y. Li, Phys. Chem. Chem. Phys. 13, 10648 (2011).

[63] S. Xiong, W. Qi, Y. Cheng, B. Huang, M. Wang, and Y. Li, Phys. Chem. Chem. Phys. 13, 10652 (2011).

[64] B. Graczykowski, A. E. Sachat, J. S. Reparaz, M. Sledzinska, M. R. Wagner, E. Chavezangel, Y. Wu, S. Volz, F. Alzina, and C. M. S. Torres, Nat. Commun. 8, 415 (2017).

[65] Z. Han and A. Fina, Prog. Polym. Sci. 36, 914 (2011), special issue on composites.

[66] X. Xu, J. Chen, J. Zhou, and B. Li, Adv. Mater. 30, 1705544 (2018). 